\title{
Handling Low-Density LiDAR Data: Calculating the Heights of Civil Constructions and the Accuracy Expected
}

\author{
Rubén Martínez Marín, Elena Lianes Revilla, Juan Carlos Ojeda Manrique, and \\ Miguel Marchamalo Sacristán \\ Laboratorio de Topografía y Geomática, Departamento de Ingeniería y Morfología del Terreno, E.T.S.I. Caminos, Canales y Puertos, \\ Universidad Politécnica de Madrid, Profesor Aranguren s/n, 28040 Madrid, Spain
}

Correspondence should be addressed to Rubén Martínez Marín; ruben.martinez@upm.es

Received 19 September 2012; Revised 12 December 2012; Accepted 18 December 2012

Academic Editor: John Mander

Copyright (C) 2013 Rubén Martínez Marín et al. This is an open access article distributed under the Creative Commons Attribution License, which permits unrestricted use, distribution, and reproduction in any medium, provided the original work is properly cited.

\begin{abstract}
During the last years, in many developed countries, administrations and private companies have devoted considerable amounts of money to obtain mapping data using airborne LiDAR. For many civil activities, we can take advantage of it, since those data are available with no cost. Some important questions arise: Are those data good enough to be used for determining the heights of the civil constructions with the accuracy we need in some civil work? What accuracy can we expect when using low-density LiDAR data $\left(0.5 \mathrm{pts} / \mathrm{m}^{2}\right)$ ? In order to answer those questions, we have developed a specific methodology based on establishing a set of control points on the top of several constructions and calculating the elevation of each one using postprocessing GPS. Those results have been taken as correct values and the comparison between those values and the elevations obtained, assigning values to the control points by the interpolation of the LiDAR dataset, has been carried out. This paper shows the results obtained using low-density airborne LiDAR data and the accuracy obtained. Results have shown that LiDAR can be accurate enough (10-25 $\mathrm{cm})$ to determine the height of civil constructions and apply those data in many civil engineering activities.
\end{abstract}

\section{Introduction}

LiDAR (light detection and ranging) system is a well-known technology to acquire mapping information. Efficiency and affordability have made LiDAR a primary tool for collecting a variety of high-quality surface data in much shorter periods of time than previously possible. In addition, hardware LiDAR technology has been significantly improved. Furthermore, the ranging accuracy improved to $2-3 \mathrm{~cm}$ level, and the availability of intensity signal became common [1]. These developments resulted in improved data quality in terms of higher point density and better accuracy, which, in turn, opened new application areas of LiDAR [2]. Modern LiDAR systems with the $\mathrm{cm}$ level ranging accuracy and high pulse rate, in theory, could be applied on topography works, like leveling processes [3], even more, ground-based LiDAR systems are being used to monitor movements of large structures and landslides, as a complement of other instruments, for instance, nonprism total station [4].
However, besides the laser ranging error there are several potential error, sources that can degrade the accuracy of the acquired data. LiDAR systems are complex multisensor systems and incorporate at least three main sensors: the GPS and INS navigation sensors and the laser-scanning device (Figure 1).

There are multiple causes of errors that affect the process: navigation errors, individual sensor calibration or measurement errors, and intersensor calibration errors or a misalignment between the different sensors [5]. Those kinds of errors can be minimized applying a rigorous system calibration, but there are always discrepancies between the reality and the acquired data. Nevertheless, the accuracy achieved with this system is good enough to be applied on most of the usual civil engineering activities.

Currently, many methods have been developed to measure the accuracy of the acquired data [6-8]. The main conclusion is that the vertical accuracy is higher than the 


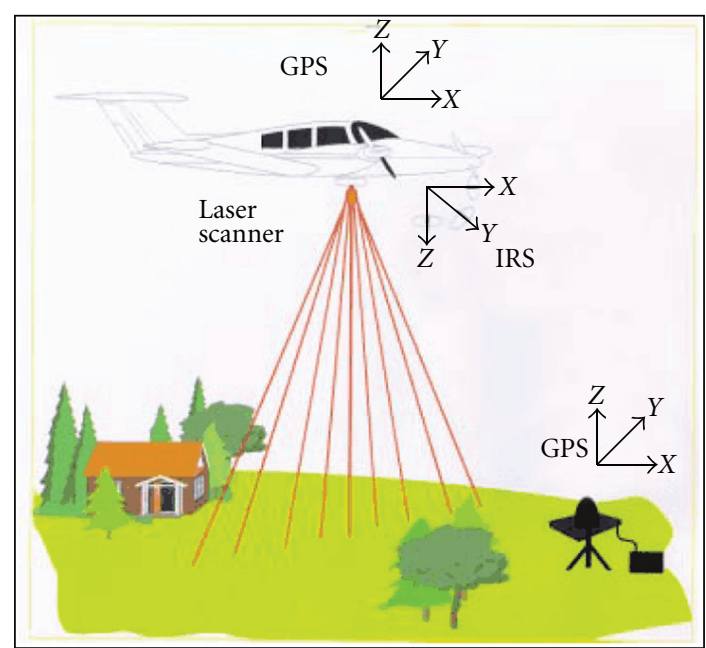

Figure 1: LiDAR system components.

TABLE 1: LiDAR accuracy.

\begin{tabular}{lcc}
\hline \multirow{2}{*}{ LiDAR point density $\left(\mathrm{pts} / \mathrm{m}^{2}\right)$} & \multicolumn{2}{c}{ Accuracy $(\mathrm{cm})$} \\
& Horizontal & Vertical \\
\hline 16 & $2-3$ & 1.3 \\
4 & $5-10$ & 2.5 \\
1.78 & $10-15$ & 4.0 \\
\hline
\end{tabular}

horizontal, in other words, horizontal errors in LiDAR data are usually more significant [9] than vertical errors.

The latest studies show that the positioning accuracies obtained, using optimal targets and different LiDAR point densities, may be from $2.0 \mathrm{~cm}$ to $15.0 \mathrm{~cm}$ [10-13]. Table 1 [10] shows the most representative values obtained.

When using a density close to $2 \mathrm{pts} / \mathrm{m}^{2}$, a good enough accuracy can be expected, $4.0 \mathrm{~cm}$ error at vertical positioning, but we are using a lower density, $0.5 \mathrm{pts} / \mathrm{m}^{2}$ (the official airbone LiDAR in Spain) without any optimal target in field; therefore, we will obtain less accuracy.

In spite of the fact that the best way to calculate elevations in civil constructions is to level the top of the object applying a geometric leveling or GPS, for some civil activities, where it is not necessary high accuracy, but the economy is very important, the use of public-data LiDAR can help to achieve good results. The main goal of this study is double, firstly, to determine whether that low-density LiDAR dataset is suitable to be used or not, to calculate the elevation, and secondly, the accuracy obtained applying the proposed methodology.

In order to carry out the research, a set of civil constructions (dams) have been selected. On the top of the dams, at least two control points have been installed and their elevations have been calculated using postprocessed GPS observations. Those elevations, obtained with GPS, are considered "correct values" and the base to compare against the elevations calculated through the LiDAR dataset.

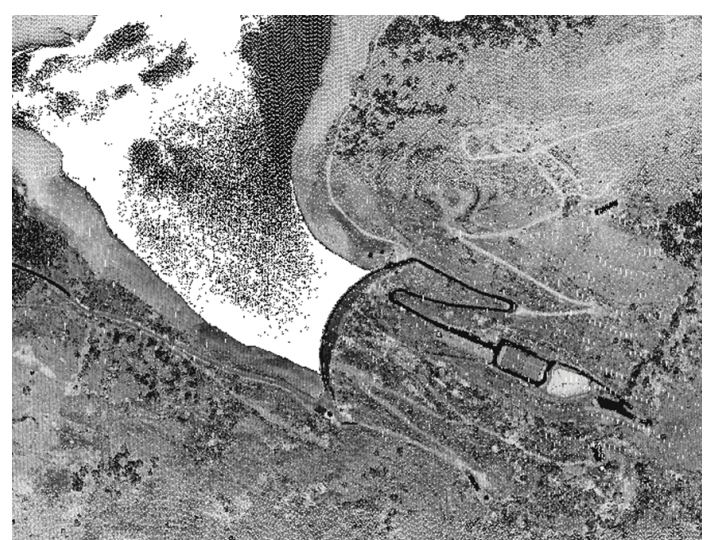

Figure 2: La Aceña Dam LiDAR points.

\section{Materials and Methodology}

2.1. LiDAR Dataset. The Spanish official LiDAR data set, the PNOA (Plan Nacional de Ortofotografía Aérea), is a costfree information system and has the following characteristics (http://www.ign.es/):

digital terrain model accuracy: RMSE $\leq 0.15 \mathrm{~m}$, density: $0.5 \mathrm{pts} / \mathrm{m}^{2}$ (low density), format file: "LAS" binary format, grid step size: $1.41 \mathrm{~m} \times 1.41 \mathrm{~m}$, coordinate system: ETRS89 (European Terrestrial Reference System 1989).

A set of thirteen significant civil constructions were selected in order to develop the current research. Firstly, at least, two reference marks were located on the top of the constructions and their elevations were calculated using a GPS system. Those elevations were considered the real height values for each construction.

Once the reference marks were built and their coordinates known, twenty-two tiles were extracted from the LiDAR data set. Each tile is a square of 2, $000 \mathrm{~m} \times 2,000 \mathrm{~m}$, containing over 2,000,000 pts (Figure 2).

2.2. Calculating "Real Coordinates". The coordinates of the reference marks, located at the crest of the constructions, were calculated applying a usual DGPS technique. The following process was repeated for each one: a GPS receiver (Leica GPS1200. Figure 3) installed on the base point, near the construction, was observed for, at least, two hours. Taking corrections from the permanent stations, coordinates of the base were calculated in the postprocessing. The rover GPS installed at the reference mark (on the top) received corrections from the static GPS located at the base point and the reference mark coordinates were calculated also in the postprocessing. Those coordinates have been considered as "real coordinates," in order to be compared with the results obtained using LiDAR data.

As the coordinates calculated with GPS are referenced to the system WGS84-ETRS89, a coordinate transformation was 
TABLE 2: LiDAR orthometric height errors.

\begin{tabular}{lccc}
\hline Reference mark $(\mathrm{Id})$ & GPS orthometric height $(\mathrm{m})$ & LiDAR orthometric height $(\mathrm{m})$ & Error $(\mathrm{m})(\mathrm{GPS}$-LiDAR) \\
\hline CN04 & 924.26 & 923.50 & $\left.0.76^{* *}\right)$ \\
BN05 & 924.35 & 924.40 & -0.05 \\
R & 907.17 & 907.25 & -0.09 \\
06 & 1318.24 & 1318.14 & $0.10^{*)}$ \\
03 & 1087.96 & 1088.04 & -0.09 \\
01 & 741.94 & 742.02 & -0.08 \\
01 & 714.82 & 714.84 & -0.02 \\
06 & 1290.30 & 1290.35 & -0.05 \\
12 & 830.41 & 830.31 & $0.10^{*)}$ \\
C05 & 725.87 & 725.90 & -0.03 \\
BN06 & 901.39 & 901.26 & $\left.0.14^{*}\right)$ \\
A & 955.63 & 955.67 & -0.04 \\
27 & 1010.83 & 1010.85 & -0.03 \\
\hline
\end{tabular}

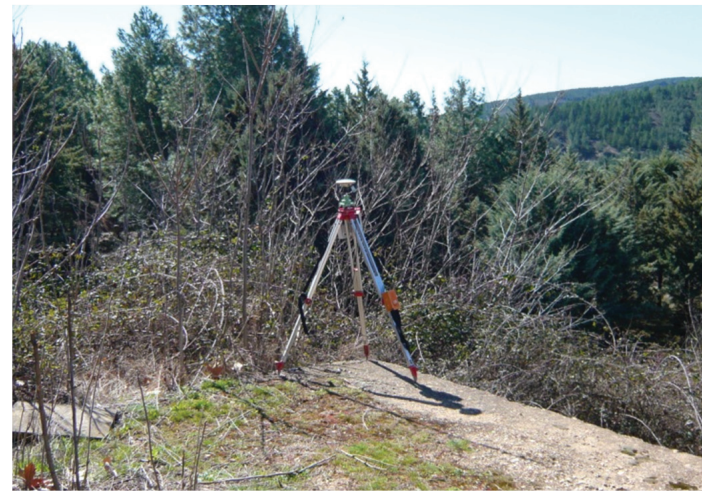

FIGURE 3: GPS receiver at the reference base near the construction.

needed in order to obtain the orthometric heights. To solve that, the official Spanish Geoid (EGM08-REDNAP), issued by the Instituto Geográfico Nacional (IGN), was applied (http://www.ign.es/).

2.3. Filtering LiDAR Data. A customized program has been developed in order to be able to select the coordinates of each topographic reference mark located on the crest of the constructions (Figure 4). That program has several possibilities as follows.

(i) Select a rectangle to rule out any point out of it.

(ii) Set up the influence radius in order to search points only in that circle.

(iii) Remove any point with a height larger than a given value.

For each construction, knowing the reference mark coordinates and using the filtering program, a subset of LiDAR points were extracted in order to obtain only points near the reference mark. In this step, the proximity criterion was applied.

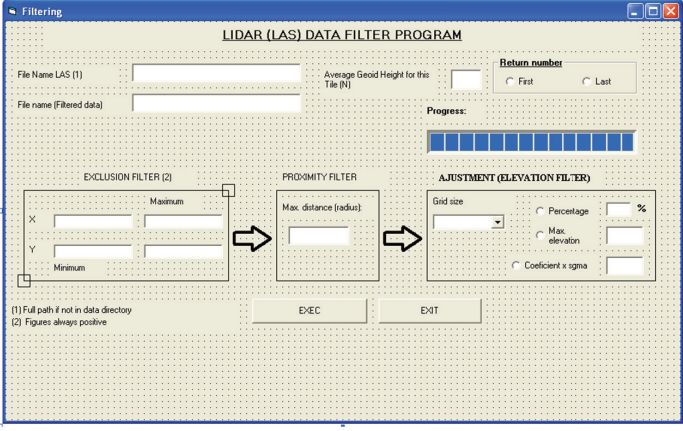

FIGURE 4: Filtering program.

2.4. Calculating Reference Mark Coordinates with LiDAR Data. Working with low-density LiDAR data is almost impossible to have LiDAR information coincident with the reference mark; therefore, a interpolating process was applied in order to estimate the $X-Y$ coordinates and then to assign the $Z$ coordinate to each reference mark. There are many algorithms that can be applied on this problem, but having so few points near the control points, the gravitational interpolation has been selected, since other techniques need more data. The procedure consisted in taking the LiDAR points around the reference mark (in $2 \mathrm{D}$, distance equal or less than $1.0 \mathrm{~m}$ ) and to apply a gravitational interpolation, to estimate the $Z$ coordinate.

Known the $X-Y$ coordinates of the reference mark, a subset of LiDAR points was extracted from LiDAR files using the filtering program. From this subset, a new selection criterion was applied.

Given a reference mark $\operatorname{RM}\left(x_{\mathrm{RM}}, y_{\mathrm{RM}}, z_{\mathrm{RM}}\right)$, being $\left(x_{\mathrm{RM}}, y_{\mathrm{RM}}, z_{\mathrm{RM}}\right)$ real coordinates obtained from GPS, a point $P\left(x_{P}, y_{P}, z_{P}\right)$ is selected when the distance from $P$ to $\mathrm{RM}$ is equal or less than $1.0 \mathrm{~m}$; that is,

$$
D_{P}^{\mathrm{RM}}=\sqrt{\left(x_{\mathrm{RM}}-x_{P}\right)^{2}+\left(y_{\mathrm{RM}}-y_{P}\right)^{2}} \leq 1.0 \mathrm{~m} .
$$


Assuming that there are $n$ points in the LiDAR subset, the estimated $Z$ coordinate, height $\left(Z_{\mathrm{RM}}^{\prime}\right)$, is given by the expression:

$$
Z_{\mathrm{RM}}^{\prime}=\frac{\sum_{i=1}^{n}\left(z_{i} /\left(D_{i}^{\mathrm{RM}}\right)^{2}\right)}{\sum_{i=1}^{n}\left(1 /\left(D_{i}^{\mathrm{RM}}\right)^{2}\right)},
$$

where $Z_{\mathrm{RM}}^{\prime}$ is the estimate $Z$ coordinate for the reference mark given, $z_{i}$ is the $Z$ coordinate of point $i, D_{i}^{\mathrm{RM}}$ is the Euclidean distance from point $i$ to RM.

\section{Results}

Table 2 shows the differences between the GPS heights and the corresponding results obtained with LiDAR. Almost all of them are negatives, that is, GPS height larger than LiDAR height, except four cases highlighted with asterisk and only one with double asterisk (higher discrepancy) that will be discussed later, in the next section.

Error was always measured by subtracting the LiDAR from the GPS elevation, resulting in positive errors for an underprediction of the orthometric height. Several measures of error were computed: mean signed error (Table 2), mean absolute error, and RMSE. The RMSE was computed by the expression

$$
\mathrm{RMSE}_{\text {Observed_LiDAR_Pts }}=\sqrt{\frac{\sum\left(Z_{\mathrm{GPS}}-Z_{\mathrm{LiDAR}}\right)^{2}}{n}}=0.22
$$

and the mean absolute error (MAE) by

$$
\mathrm{MAE}_{\text {Observed_LiDAR_Pts }}=\frac{\sum\left|Z_{\mathrm{GPS}}-Z_{\mathrm{LiDAR}}\right|}{n}=0.12 \text {. }
$$

\section{Discussion}

The primary goal in this study is to validate the LiDAR data set as a good source of information to determine the height of a construction, suitable to be applied in many civil engineering activities. The results show that the mean absolute error is less than $12.00 \mathrm{~cm}$ and the root mean square error is less than $22.30 \mathrm{~cm}$. With these values, we can affirm that it is viable to apply this methodology to determine the height; nevertheless, there are some results that are positive, while the majority are negative and small. In order to discover the reason why that happens, we had to visit the dams again, looking for the reference marks.

All figures show the same characteristic (Figures 5 and 6): the reference marks are on the sidewalk or over a concrete base, always located higher than most of the data extracted from LiDAR tiles. Since there are not LiDAR points, exactly over the reference mark, we have processed the heights of the nearest points (distance $\leq 1.0 \mathrm{~m}$ ). In case of not considering

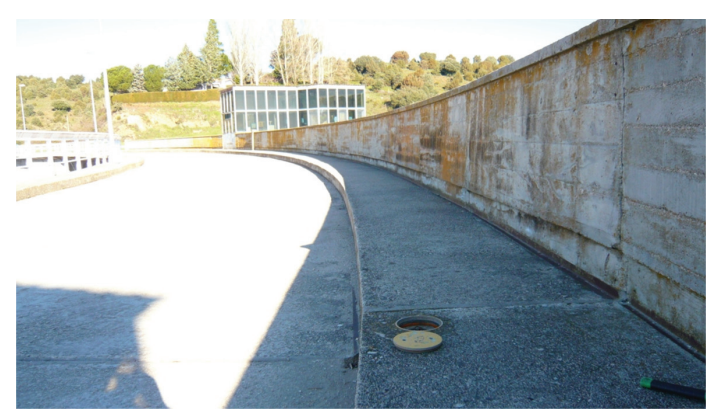

FIGURE 5: Reference mark 12.

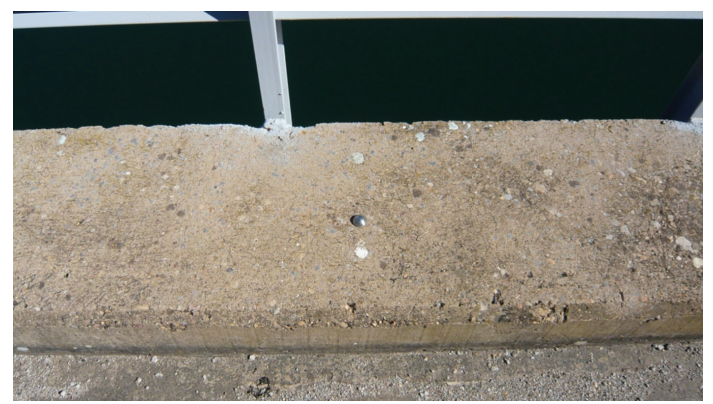

FIGURE 6: Reference mark BN06.

those values, the new errors calculated by (3) and (4) would be (being in this case $n=9$ )

$$
\begin{aligned}
\mathrm{RMSE}_{\text {Observed_LiDAR_Pts }} & =0.0558, \\
\text { MAE }_{\text {Observed_LiDAR_Pts }} & =0.050 .
\end{aligned}
$$

As we can see above, if that subset of reference marks are removed, a significant reduction of the errors is obtained.

\section{Conclusions}

The LiDAR data set produced a high-quality topographic survey of the constructions, suitable to obtain the ellipsoidal and orthometric heights (these ones after a transformation applying the geoid height). It is not necessary to install reflectors to obtain a good enough precision.

The accuracy expected depends on the LiDAR point density [14]; nevertheless, using $0.5 \mathrm{pts} / \mathrm{m}^{2}$, we have obtained around $5 \mathrm{~cm}$ discrepancy with "real coordinates" of the reference marks, assuming those as control points that were obtained by postprocessed GPS.

Starting from the raw LiDAR data set, it is not necessary to generate a digital terrain model (DTM) to calculate the elevation of the control points; even though almost current procedures use this methodology, a direct interpolation from the raw data can be applied [15].

The results obtained prove that the low-density LiDAR dataset is suitable to be applied in many civil engineering activities where an approximate height is needed and the economy factor is essential. 


\section{Acknowledgments}

The authors would like to thank the Canal de Isabel II and the Instituto Geográfico Nacional not only their contribution and help but also for providing essential information and data for this research.

\section{References}

[1] C. Toth, "Future Trends in LiDAR," in Proceedings of the ASPRS Annual Conference (CD-ROM), Denver, Colo, USA, May 2004.

[2] M. Renslow, "The status of LiDAR today and future directions," in 3D Mapping from InSAR and LiDAR, ISPRS WG I/2 Workshop (CD-ROM), Banff, Canada, June 2005.

[3] H. Whenquan, The International Archives of the Photogrammetry, Remote Sensing and Spatial Information Sciences, vol. 37, Part B4, 2008.

[4] I. T. Yang, J. K. Park, and D. M. Kim, "Monitoring the symptoms of landslide using the non-prism total station," KSCE Journal of Civil Engineering, vol. 11, no. 6, pp. 293-301, 2007.

[5] E. P. Baltsavias, "Airborne laser scanning: basic relations and formulas," ISPRS Journal of Photogrammetry and Remote Sensing, vol. 54, no. 2-3, pp. 199-214, 1999.

[6] H. Burman, "Laser strip adjustment for data calibration and verification," International Archives of Photogrammetry and Remote Sensing, vol. 34, Part 3A, pp. 67-72, 2002.

[7] S. Filin, "Analysis and implementation of a laser strip adjustment model," International Archives of Photogrammetry and Remote Sensing, vol. 34, Part 3/W13, pp. 65-70, 2003.

[8] C. Toth, N. Csanyi, and D. Grejner-Brzezinska, "Automating the calibration of airborne multisensory imaging systems," in Proceedings of the ACSM-ASPRS Annual Conference (CDROM), April 2002.

[9] G. Vosselman and H. G. Maas, "Adjustment and filtering of raw laser altimetry data," in Proceedings of the OEEPE Workshop on Airborne Laserscanning and Interferometric SAR for Detailed Elevation Models, OEEPE Publications no. 40, pp. 62-72, 2001.

[10] N. Csanyi, C. Toth, and D. Grejner-Brzezinska, "Using LiDARspecific ground targets: a performance analysis," in ISPRS WGI/2 Workshop on 3D Mapping from InSAR and LiDAR (CDROM), Alberta, Canada, 2005.

[11] N. Csanyi, C. Toth, D. Grejner-Brzezinska, and J. Ray, "Improving LiDAR data accuracy using LiDAR-specific ground targets," in ASPRS Annual Conference (CD-ROM), Baltimore, Md, USA, March 2005.

[12] N. Csanyi and C. Toth, "On using LiDAR-specific ground targets," in ASPRS Annual Conference (CD-ROM), Denver, Colo, USA, May 2004.

[13] W. Xiaohui et al., "Precision tests LiDAR data products," in Engineering of Surveying and Mapping, pp. 67-69, 2007.

[14] G. Sohn and I. Dowman, "Data fusion of high-resolution satellite imagery and LiDAR data for automatic building extraction," ISPRS Journal of Photogrammetry and Remote Sensing, vol. 62, no. 1, pp. 43-63, 2007.

[15] N. Karacic and R. Marshall, "Vertical component of topographic survey Vs. SRTM, ICEsat, and NED elevations for dallas levees project area," in ACSM/ESRI Survey Summit Paper SUR 1073, 2012. 

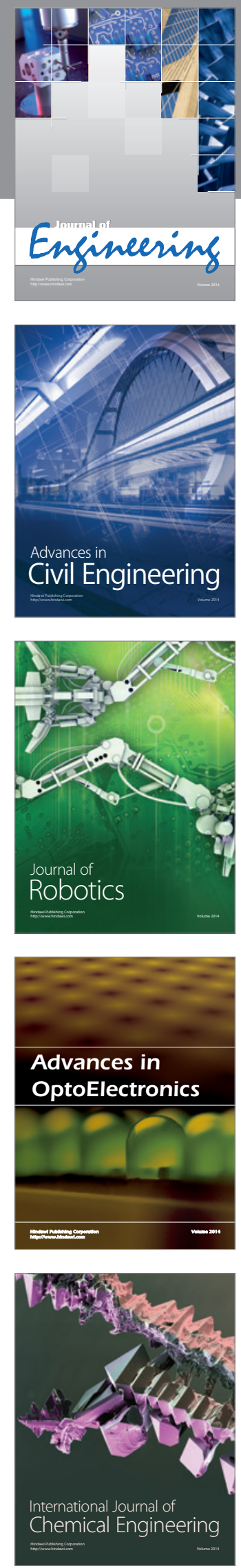

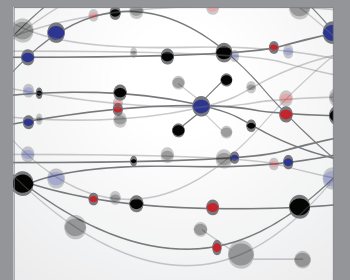

The Scientific World Journal
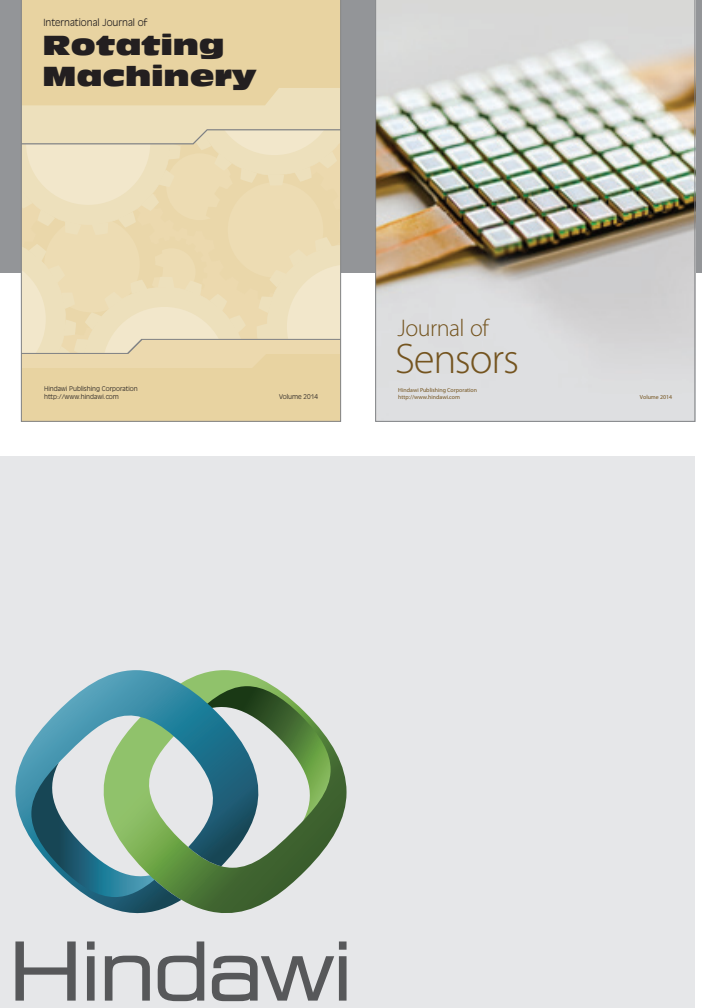

Submit your manuscripts at http://www.hindawi.com
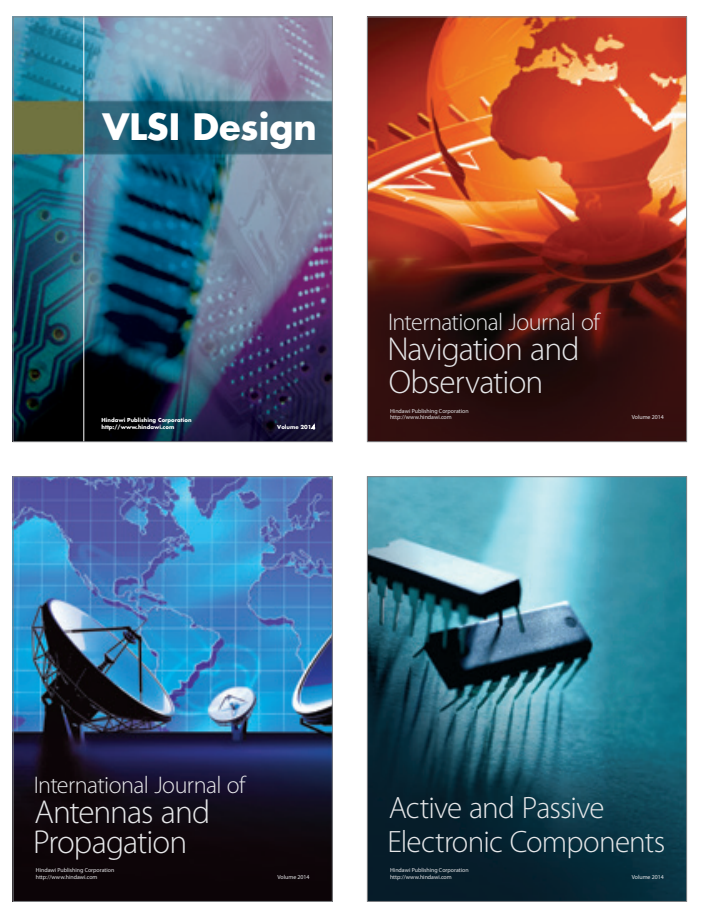
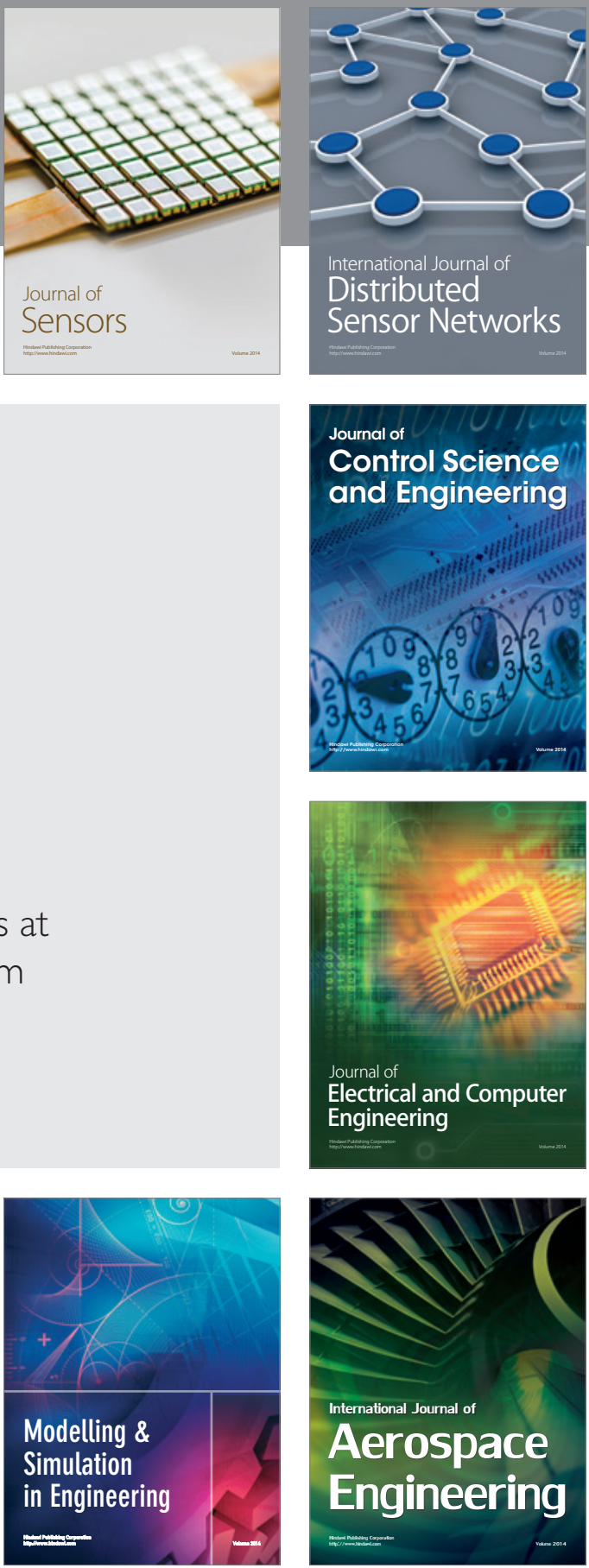

Journal of

Control Science

and Engineering
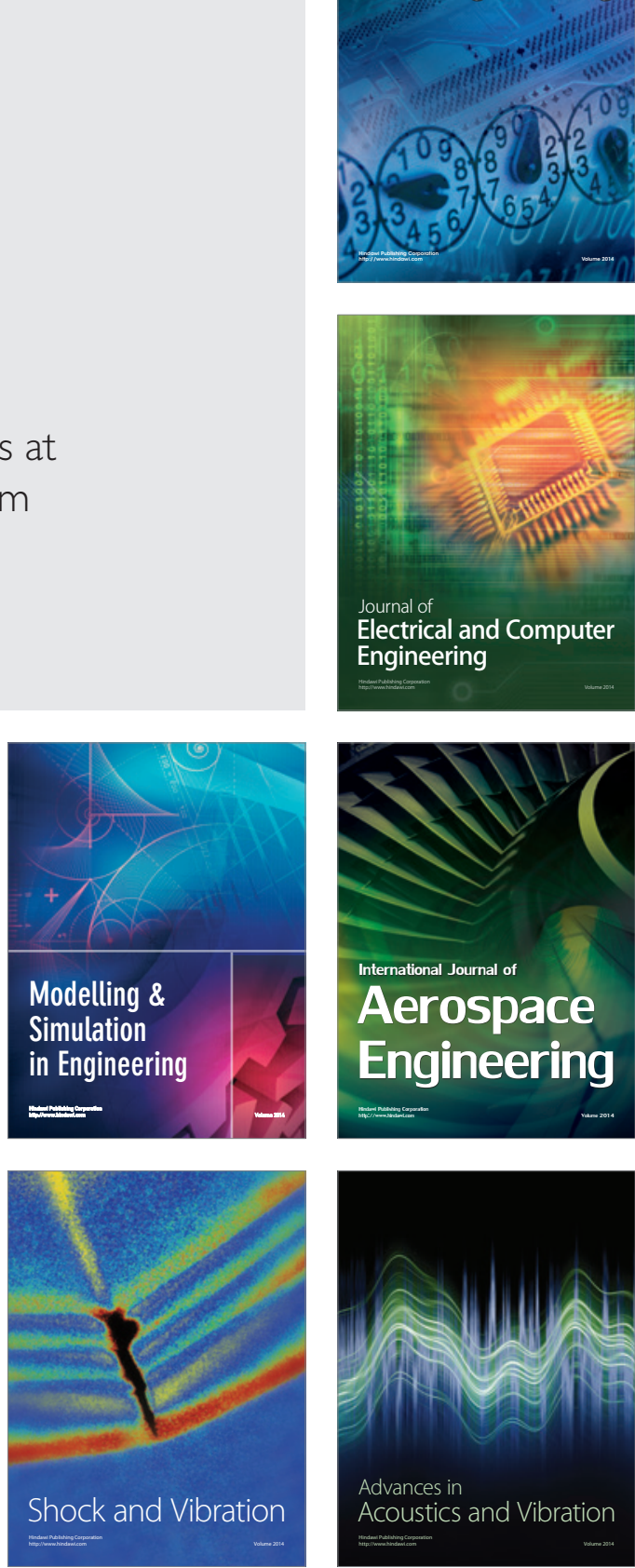